\title{
REDUCING MORTALITY FROM PERFORATED PEPTIC ULCER IN NEPAL
}

Strugnell N A

\section{ABSTRACT}

A study was undertaken to compare mortality from perforated peptic ulcer before and after introduction of pre-operative, intra-operative and post-operative measures to improve clinical care of patients affected by this condition. Data was accrued prospectively over 6 months (24 patients) and compared with the previous 6 months of historical controls (27 patients). Mortality showed a trend towards improvement. (9/27 c.f. 3/ 24, $\mathrm{p}=\mathbf{0 . 1 2}$ n.s., Fisher exact test). Based on these findings and changing ideas in regard to disease transmission emerging from the recent literature, a framework for limiting mortality from perforated peptic ulcer in Nepal is proposed.

\section{Key Words: Perforation, peptic ulcer, Nepal, mortality reduction, Helicobacter pylori.}

\section{INTRODUCTION}

In the surgical department at United Mission Hospital Tansen (UMHT) morbidity and mortality data are audited fortnightly. At the routine 6 monthly mortality review in February 2000, it was evident that complications of benign duodenal or gastric ulcer disease were the commonest single diagnosis leading to mortality of patients admitted to UMHT under the care of the surgical department $(15 / 50,30 \%)$. The majority of these patients had experienced perforation of a peptic ulcer.

It was postulated that patients died from one or more of three pathophysiological mechanisms: hypovolaemic and or septic shock (often receiving inadequate pre-operative and intraoperative attention), post-operative hypoxic respiratory failure with or without associated sepsis and late sepsis due to advanced peritonitis at the time of presentation.

At a multi-disciplinary mortality review meeting strategies for

Address for correspondence : $\quad$ Dr. Neil A. Strugnell

Department of Surgery

United Mission Hospital, Tansen

UMN

P.O. Box: 126, Kathmandu, Nepal.

Email: strugnel@umn.org.np reducing in-hospital mortality from this disease were discussed. Key concepts for mortality reduction were then disseminated broadly so that all hospital staff involved in treatment of these patients were able to participate in an effort to reduce mortality. It was decided to undertake a study to determine whether these improvements in clinical care were making any impact upon mortality from peptic ulcer perforation.

\section{MATERIALS AND METHODS}

Patients having laparotomy for treatment of perforated duodenal or benign gastric ulcer were included in the study even if the initial laparotomy had been performed elsewhere. Patients with bleeding ulcer, malignant ulcer or peritonitis from unknown cause or cause other than perforated peptic ulcer were excluded from the study. Patients who died of peritonitis without laparotomy were also excluded.

Hospital medical records were used to obtain patient, disease 
and operation related data, surgical, anaesthetic and post-

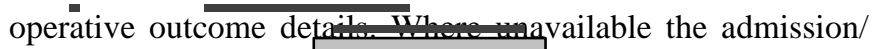
discharge database w: $\quad$ mortality data and the operation room regi o obtain disease and operation details. $\mathrm{s}$ obtained from the cashier's records. Cos en per life saved were

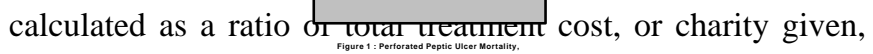
for the cohort (Groupler ? d divinided by the number of survivors from the cohort.

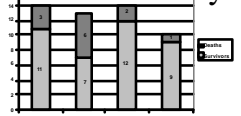

Data was collected retrospectively for the historical controls fron month study block (Group 1) and pros $\quad$ es from the latter 6 month study block (Gro

Operative method was by simple omental plug closure and peritoneal toilet, only, in the majority of the patients.

The pre-operative, intra-operative and post-operative interventions (i.e.: improvements in clinical care) that were introduced in the second half of the study are outlined in the discussion section.

Statistical analysis for age comparisons was done by employing the Mann-Whitney U-test. Mortality and all other variables were analysed by using Fisher's Exact test. $\mathrm{P}$ values $<0.05$ were considered to be statistically significant.

\section{RESULTS}

There were no significant differences in patient, disease or operation related characteristics between the two study groups. The median age of patients in Group 2 was non-significantly older by 6.5 years.
Figure 1. Histogram showing deaths and survivors as a proportion of total cases within consecutive 3 month blocks. Blocks A and B correspond to Group 1 patients, blocks $C$ and $D$ represent the Group 2 patients. Group E represents an incomplete 3 month block with no mortality immediately following the end of the 12 month study period.

In Figure 1, the trend towards decreasing mortality from perforated peptic ulcer at UMHT over and beyond the study period can be clearly seen.

In the first 6 months (Group 1) almost half of the deaths occurred in patients equal to or younger than the median age of 42 years. Ages of patients who died from Group 1 are 20, $21,35,42,50,60,65,69$, and 75 years. In the latter 6 months (Group 2) no deaths occurred in patients younger than the median age for the group, 48.5 years. Ages of patients who 
died from Group 2 are 50, 65 and 67. These findings, in addition to the finding that patients from Group 2, who, despite being of 6.5 years older median age than those in Group 1, showed a non-significant reduction in mortality $(\mathrm{p}=0.12)$, provide further corroborative evidence of improved mortality outcomes in the latter half of the study.

Deaths of patients from Group 1 were evenly distributed between early Day 0 (2), Day 1 (2), Day 3 (1) and late Day 4 (1), Day 6 (1), Day 11 (1) and Day 19 (1). Of those dying from Group 2 deaths occurred early Day 1 (1) and day 3 (2).

The need for re-laparotomy was equally shared between the two groups (3 each). Of patients who required re-laparotomy they tended to have re-operation earlier and improved outcome if in Group 2 (Group 1 re-laparotomy mortality $2 / 3$ c.f. Group $2,0 / 3)$. Intra-abdominal abscess was found in four of the six patients who required re-laparotomy.

Re-laparotomy had a significant impact on treatment cost. The mean cost of treatment per patient averaged across the whole study (survivors and non-survivors included) was 14,687 Nepali Rs. Of the six patients requiring re-laparotomy the mean treatment cost per patient was more than doubled 35,400 NRs.

\section{DISCUSSION}

Reduction in mortality from perforated peptic ulcer in Nepal can essentially be achieved by a combination of three mechanisms:

a) improved clinical care of patients presenting to hospital with this disease,

b) improving access to surgical facilities where such care can be provided and

c) taking measures to reduce the prevalence of peptic ulcer perse.

The first aspect of "improved clinical care" is the ongoing critical analysis of medical services by means of audit. It is encouraging to observe the emphasis that this journal has recently placed on audit activities ${ }^{1}$. Ongoing audit of the work of the surgical department at UMHT enabled us to identify perforated peptic ulcer as a key disease warranting further research and protocol development.

It is difficult to elucidate precisely which improvement in practice lead to the observed reduction in mortality from peptic ulcer perforation. However, it is likely that a combination of improved practices have contributed to the mortality reduction. The following is an outline of the improvements in care that were used in the second part of the study. These principles form the basis for our current treatment protocol.

\section{Improved Pre-Operative Care}

The main pre-operative factor requiring optimisation prior to operation for perforated peptic ulcer is adequate pre-operative fluid resuscitation. Previously we had a policy that if less than $0.5 \mathrm{ml} / \mathrm{kg} /$ hour of urine was produced by the patient then laparotomy would be deferred (as attempts to perform operation in the setting of inadequate rehydration are associated with an unacceptably high mortality rate). The formula, although technically correct, contributed to a scenario whereby patients were stranded on the wards receiving inadequate resuscitation whilst surgeons and anaesthetists were occupied elsewhere. During the second half of this study we attempted to promptly transfer these critically ill patients to the anaesthetic preoperative/ post-operative area where anaesthetists and surgeons could actively participate in the patient's resuscitation. After introduction of the new protocol IV cannulae 14 or 16 gauge were inserted bilaterally. Resuscitation with $2.5-3.5 \mathrm{~L}$ of Ringer's Lactate or Normal Saline over 2-3 hours was then commenced. A Foley catheter was routinely inserted and a urine output of $1 \mathrm{ml} / \mathrm{kg} /$ hour was aimed for. However, generally laparotomy was proceeded to if urine output had exceeded $0.75 \mathrm{ml} / \mathrm{kg} /$ hour. The downward spiral of septic shock could therefore be broken at the earliest possible opportunity for intervention. In addition this enabled the post-operative recovery to occur before the late evening when staffing levels were lower and when staff fatigue may have exerted a negative impact on outcome.

Placement of a nasogastric tube with pre-operative decompression by aspiration and administration of broad spectrum antibiotics (Gentamicin, Ampicillin and Metronidazole) and Ranitidine intravenously also formed part of the pre-operative routine.

\section{Improved Intra-Operative Care}

Laparotomy was performed through an upper midline incision. An omental plug closure technique without prior closure of the perforation was the method favoured by the author but there was some variation amongst the surgeons regarding actual closure technique. Residents performing the operation under supervision were reminded that they should use a wellvascularised, tension-free omental plug and that adequate bites of healthy tissue $(>0.7 \mathrm{~cm})$ away from the edge of the perforation need to be taken. Sutures across the omental plug should not be applied with excessive tension.

The biggest intra-operative change in emphasis was the need to assiduously search for intraperitoneal collections. This was done using an anatomical method whereby all potential areas that pus may have collected were systematically sought then drained i.e.: right and left subphrenic spaces, subhepatic space, right and left paracolic gutters, pelvis and between bowel loops. 
If present, collections were drained and irrigated with warmed normal saline until the effluent became clear. In general drains were not used except where there was bleeding following drainage of chronic vascularised collections. Surgeons were encouraged to call a colleague to assist in difficult cases, especially in late peritonitis, where the exclusion of interloop abscesses demanded careful lysis of vascular adhesions between bowel loops. Attention to detail with regard to peritoneal toilet not only contributed to mortality reduction but had the added advantage of reducing the need for re-laparotomy (and its attendant doubling of the total treatment cost).

The abdomen was closed using a tension free continuous mass closure technique with a 1 polydiaxonone sulfate suture. We favour the use of subcutaneous bupivicaine infiltration $(40 \mathrm{ml}$ of $0.25 \%$, half life $4-6$ hours) in order to enhance post-operative ventilation and facilitate chest physiotherapy.

\section{Improved Post-Operative Care}

The greatest change in post-operative care was an emphasis on the importance of preventing respiratory complications. Patients were positioned $35^{\circ}$ head up in order to decrease the work of breathing. They were prescribed regular parenteral analgesia (morphine $0.1 \mathrm{mg} / \mathrm{kg} 4$ hourly IM), rather than prn analgesia. This reduced atelectasis by enhancing deep respiration and co-operation with chest physiotherapy exercises.

Oxygen was delivered at a rate of $3 \mathrm{~L} /$ minute via nasal prongs for the first 24 hours and $2 \mathrm{~L} /$ minute nasal prongs for the second 24 hours post-operatively. Some patients had lower or higher oxygen requirements which were determined clinically or with the adjunctive use of oximetry which became available late in the study.

All patients received regular chest physiotherapy and were encouraged to ambulate early and sit out in a padded chair on the first post-operative day. We emphasised that physiotherapy was a prescribed treatment which if omitted would lead to complications such as pneumonia and bedsores. As physiotherapists are not always on duty, doctors and nurses also needed to share the responsibility of encouraging breathing exercises and early mobilisation.

Doctors caring for these critically unwell patients were encouraged to review these patients more than once daily. Fluid, analgesia and oxygen therapy need to be optimised. In addition doctors need an index of suspicion that there may be retained collections of pus that may warrant re-laparotomy.

The incidence of nosocomial pneumonia is known to be higher in the presence of a nasogastric tube so these were generally removed on the second post-operative day. Likewise, urinary catheters were removed on the second post-operative day, if patients were showing signs of recovery, in order to limit the risk of sepsis. Free oral fluids were usually commenced on the $3^{\text {rd }}$ post-operative day and at that stage definitive treatment of the peptic ulcer with Omeprazole $20 \mathrm{mg} \times 2 \times 42$ days, Metronidazole 400mg x 3 × 14 days and Amoxycillin 500mg x 3 x 14 days was commenced. This both ensured healing of a complicated ulcer (6 weeks proton pump inhibitor therapy) and eradicated Helicobacter pylori with a greater than $85 \%$ probability ${ }^{2}$. Thorough explanation of drug treatment is critical to compliance. The prescription of more than one medicine, especially amongst illiterate patients, has been shown to be associated with decreased patient understanding, with anticipated poor compliance ${ }^{3}$. A translator was used if the patient's first language was not Nepali.

The careful application of the above principles enabled us to reduce mortality from perforated peptic ulcer at UMHT.

Whilst we are pleased with the mortality reduction achieved for perforated peptic ulcer at UMHT, it is not feasible nationally to reduce mortality from this disease by relying on a small number of institutions. This study shows that for both groups there was a median interval of 3 days between perforation and presentation to hospital. This indicates that there are an inadequate number of local facilities catering for a poor patient population that are capable of providing surgical care. This compares very unfavourably with hospital services in metropolitan areas where the time between perforation and presentation may be in the order of 1- 6 hours. Early peritonitis is much simpler to deal with surgically as free debris within the peritoneal cavity is easily dealt with by lavage and suction. However, as time elapses prognosis deteriorates ${ }^{4,5}$ as a dense fibrinous reaction develops and localised collections form. These abscess cavities later become vascularised and fibrinous adhesions between loops of bowel form. In the later stages attempts to divide these adhesions are associated with a higher risk of visceral injury, leak and fistula formation. However, the consequences of ongoing sepsis from missed interloop abscesses where a careful dissection is not performed are also likely to be fatal.

At UMHT we have been participating for several years in the MDGP training program. Trainees learn general surgical skills whilst on rotation to the Surgical Department. Those who continue surgical practice as a significant component of their work will be capable of performing laparotomy for perforated peptic ulcer where peritonitis has not progressed to a late stage. Mortality from perforated peptic ulcer could be reduced in Nepal if patients, the majority of whom are rural-dwellers, were able to access hospitals where basic general surgery was 
performed by GP surgeons. However, it will take more than trained GP surgeons to achieve this goal. Local hospitals would need to provide infrastructure so that basic emergency surgery could be performed. This should include a regular supply of nurses, anaesthetists and surgically skilled GP's who could perform surgery on a daily basis. If surgery continues to remain the domain of specialists surgery will continue to remain inaccessible for the majority of the rural poor. It is a vision of our institution that MDGP doctors trained in surgery and other areas will be able to use these skills whilst working in district hospitals to the benefit of rural people throughout Nepal.

It is self-evident that reducing mortality from complications such as perforation could be achieved if the prevalence of peptic ulcer disease itself could be reduced. How to do this is problematic. Options include:

a) increasing diagnosis and treatment of confirmed cases,

b) increased treatment of dyspeptic patients without confirming diagnosis,

c) limiting the risk factors known to cause the disease.

The gold standard for diagnosis of peptic ulceration is endoscopy. Barium meal can help with chronic cases but is neither sensitive nor specific. Urea breath tests, using the ${ }^{13} \mathrm{C}$ radioisotope, and serological tests will indicate infection with the Helicobacter pylori organism but do not indicate whether peptic ulcer disease is present. These tests are too expensive for widespread use and should be restricted to the confirmation of Helicobacter pylori eradication in proven cases. Endoscopy is a resource which is very difficult for poor people to access. Attempting to run endoscopy units at low cost so that they are accessible to poor patients is a formidable challenge and is not a realistic solution when dealing with such a prevalent disease.

Medical treatment with a proton pump inhibitor such as Omeprazole and antibiotics such as Amoxicillin, Metronidazole and Clarithromycin is effective in both eradication of Helicobacter pylori and cure of benign ulcer. However, although peptic ulcer is a common disease in rural Nepal, it probably accounts for only a minority of patients who experience dyspepsia. The use of anti-Helicobacter pylori therapy in the treatment of patients with uninvestigated chronic dyspepsia is controversial in the industrialised world and literature dealing with this question in developing countries is sparse. Advocating widespread treatment of all undiagnosed dyspeptics with triple therapy regimens would only serve to transfer the economic benefits of treatment from patients to pharmaceutical manufacturers. It would be much better to invest resources into creating an environment where known risk factors for the disease are minimised.
At UMHT an outpatient treatment protocol for chronic uninvestigated dyspepsia (in press) has been developed. This is an extremely prevalent problem amongst patients presenting to this hospital. First-line treatment is given with antacids, anti-parasitic (tinidazole) and anti-helminthic (mebendazole) treatment, unless clinical features such as weight loss, vomiting or gastro-intestinal bleeding indicate the need to divert to earlier investigation to exclude development of malignancy or other serious pathology. Re-presenters receive triple therapy for the eradication of Helicobacter pylori. Patients re-presenting after triple therapy are referred for endoscopy or ultrasound if appropriate. Unfortunately it is still too early to assess the longterm outcome of this protocol. There are three proven aetiological factors for peptic ulcer development:

a) smoking

b) the Helicobacter pylori organism and

c) non-steroidal anti-inflammatory drugs.

There is good evidence that cigarette smoking contributes to the development of peptic ulceration. Mechanisms include: alteration of blood flow and motility, impairment of the mucosal defence barrier, stimulation of acid secretion, induction of bile reflux, reduction of prostaglandins and delayed healing ${ }^{6}$. Smokers are also more susceptible to Helicobacter pylori infection ${ }^{7}$. It is easier to blame patients affected by smoking related conditions than to impact smoking patterns in population. Strong lobby groups are needed in Nepal to protect the poorly educated general public from the tobacco industry's unmitigated marketing efforts. The activities of targeting developing countries in marketing this harmful product are well known'. It is encouraging to see the lead being taken by some South Asian neighbours in controlling this menace to the community's health. ${ }^{9}$

The association between peptic ulcer and the spiral organism Helicobacter pylori was first confirmed in 1985 by Marshall et al. ${ }^{10}$ This organism resides in the gastric antrum and on islands of gastric metaplasia in the duodenum ${ }^{11}$. The organism produces a urease enzyme so by conversion of urea to alkaline ammonia the organism can survive in the acid environment of the stomach. Ulcer pathogenesis is by production of proinflammatory factors and by local humoral influences. Many strains produce factors directly causing inflammation such as the Cag A protein and a vacuolating cytotoxin ${ }^{12}$. In addition, strains with the picB gene are capable of inducing the cytokine interleukin-8 which causes inflammation and injury ${ }^{13}$. Important humoral influences include raised acid output by increasing local gastrin production ${ }^{14,15}$ and by reducing somatostatin production ${ }^{15}$. 
Although Helicobacter pylori has been found in cats ${ }^{16}$ and sheep,${ }^{17}$ the worldwide incidence strongly supports man as the reservoir for infection. ${ }^{16}$ There is emerging evidence for the faecal-oral route as the actual mode of transmission for this disease. Helicobacter pylori has been both cultured from faeces ${ }^{18}$ and found in faeces using polymerase chain reaction techniques ${ }^{19}$ so water contamination may well be the source of the disease. A recent study from Central Europe ${ }^{20}$ confirmed by PCR techniques that Helicobacter pylori can be present in well water supplies. Their cohort study concluded that drinking well water was a significant risk factor for development of Helicobacter pylori infection. In addition to water supply problems, low socioeconomic status, overcrowded housing, low educational achievement of parents and rural location have also been shown to be risk factors for the disease. ${ }^{18,21,22}$

To date there have been no seroprevalence studies of Helicobacter pylori infection in Nepal. Studies from India confirm a population seroprevalence rate in the order of $80 \%$ and confirm increasing prevalence with lower socioeconomic status. $^{23,24}$ A study from Bangladesh ${ }^{25}$ showed an overall prevalence rate of $83 \%$ (68\% in children, $87 \%$ in adults). Infection rates increased from $47 \%$ in the $1-4$ aged group to over $96 \%$ for those aged greater than 50 years. In this study there were slightly higher prevalence rates in urban compared to rural dwellers. However, the relationship between low income and low educational achievement with increased prevalence rates was again confirmed.

\section{CONCLUSION}

Peptic ulcer perforation is a potentially fatal disease. Mortality from this disease (and treatment cost per life saved) can be reduced with improvements in hospital care. Of these, attention to pre-operative fluid resuscitation, intra-operative peritoneal toilet and post-operative respiratory care are probably the most important.

Late peritonitis will continue to have a high mortality rate. However, the provision of a greater number of basic surgical facilities, accessible to Nepal's largely rural population, where early peritonitis can be treated on an emergency basis, will reduce mortality from this disease.

\section{ACKNOWLEDGMENTS}

Thanks to fellow surgeons at UMHT for allowing their cases to be included in this study. I am grateful to Rajendra Nepali for histogram, Paul Kitchen and Michael Henderson for assistance with statistics, Anne Casey for accessing references and Angus McKellar for proofreading.

\section{REFERENCES}

1. Shama S. Role of Auditin Obstetrics and other specialties.J Nepal Med Assoc. 2000; 39(135): I-II

2. WalshJ H, Peterson WL. The treatment of Helicobacter pylori infection in the managementof peptic ulcerdisease. NewEng. J Med 1995; 333: 984-91

3. McKellar A. (Unpublished data). Factors affecting patient understanding of prescribed drug therapy in Westem Nepal. UMHT, 2000.

4. Bohnen J, BoulangerM, Meakins J L, McLeanAPH. Prognosis in generalized peritonitis: relation to cause and risk factors. Arch Surg 1983; 118: 285-90

5. PitcherWD, MusherDM. Critical importanceof early diagnosis and treatmentof intra-abdominal infection. Arch Surg 1982; 117: $328-33$

6. Muller-LissnerSA. Bile refluxis increased incigarettesmokers. Gastroenterology 1986; 90: 1205-09

7. Bateson MC. Cigarette smoking and Helicobacter pylori infection.

Postgrad MedJ 1993; 69: 41-44

8. United Nations Research Institute for Social Development States in Disarray. UNRISD Report 1995, Geneva.

9. Gupta PC. Tobacco Products Bill 2001: Anaid to public health. The NatMed J of India 2001; 14(3): 131-34

10. Marshall BJ , McGechie DB, Rogers PA, Glancy RJ . Pyloric Campylobacter infection and gastroduodenal disease. Med] Australia 1985; 142: 439-44

11. Goodwin CS. Duodenal ulcer, Campylobacter pylori and the "leaking roof" concept Lancet 1988; 2: 1467-69

12. Goodwin CS, Mendall MM, NorthfieldTC. Helicobacter pylori infection. Lancet 1997; 349: 265-69

13. Tummuru MKR, SharmaSA, Blaser MJ . Helicobacter pylori picB, a homologue of the Bordetella pertussis secretion protein, is required for induction of IL-8 in gastric epithelial cells. Molec Microbiol 1996; 18: 867-76

14. Levi S, Beardshall K, Haddad G etal. Campylobacter pylori and duodenal ulcers: the gastrin link. Lancet 1989; 1: 116768

15. Calam J. Helicobacter pylori, acid and gastrin. Eur J Gastroenterol Hepatol 1995; 7: 310-17

16. Bourke B, J ones N, Sherman P. Helicobacter pylori infection and peptic ulcer disease in children. Pediat Inf DisJ 1996; 15: $1-13$

17. Dore MP, Sepulveda AR, El-Zimaity HMT etal. Isolation of Helicobacter pylori from sheep: Implications fortransmission to humans. Gut (Epidemiology and Transmission Conf. Abstracts) 2000; A46: 6/5 
18. Mendall MA, NorthfieldTC. Transmission of Helicobacterpylori infection. Gut 1995; 37: 1-3

19. Makristhasis A, Pasching E, Schutske K etal. Detection of Helicobacter pylori in stool specimens by PCR and antigen enzyme immunoassay.J Clin Microbiol 1998; 36: 2772-74

20. Krumbiegel $P$, Boeckler D, Lehmann I etal. Is water a source of Helicobacter pylori infection in Central Europe? Gut (EpidemiologyandTransmissionConf. Abstracts)2000;A46: $6 / 6$

21. FiederekSC, MalatyHM, Evans DI, Pumphery CL. Factors influencing the epidemiology of Helicobacter pylori infection in children. Paediatrics 1991; 88: 578-82

22. Salomaa A, Kosunen TU, Aromaa A etal. Childhood home and H. pylori infection. Gut(Epidemiology and Transmission Conf. Abstracts) 2000; A48: 6/15
23. GrahamDY, AdamE, Redy GT etal. Seroepidemiology of $\mathrm{H}$. pylori infection in India. Comparison of developing and developed countries Dig Dis Sci 1991; 36: 1084-86

24. Peeters M, Perri F, Ghoos Y etal. Helicobacter pylori infection in school children related to socioeconomic status - multi centre study. Gut 1995; 37: Suppl. 1/A11

25. Owen RJ , NessaJ , DrasarB, ChartH. Pattems of Helicobacter pylori infection in households in Bangladesh determined by immunological status. Gut(E pidemiology and Transmission Conf. Abstracts) 2000; A49: 6/20. 